\title{
Anomalous dynamic arrest in a mixture of large and small particles
}

\author{
Angel J. Moreno ${ }^{1, *}$ and Juan Colmenero ${ }^{1,2,3}$ \\ ${ }^{1}$ Donostia International Physics Center, Paseo Manuel de Lardizabal 4, 20018 San Sebastián, Spain \\ ${ }^{2}$ Departamento de Física de Materiales, Universidad del País Vasco (UPV/EHU), Apartado 1072, 20080 San Sebastián, Spain \\ ${ }^{3}$ Unidad de Física de Materiales, Centro Mixto CSIC-UPV, Apartado 1072, 20080 San Sebastián, Spain
}

(Received 6 April 2006; revised manuscript received 17 July 2006; published 21 August 2006)

\begin{abstract}
We present molecular dynamics simulations of the slow dynamics of a mixture of large and small soft spheres with a large size disparity. The dynamics are investigated in a broad range of temperature and mixture composition. As a consequence of the large size disparity, large and small particles exhibit very different relaxation times. As previously reported for simple models of short-ranged attractive colloids and polymer blends, several anomalous dynamic features are observed: (i) sublinear behavior for mean-squared displacements, (ii) concave-to-convex crossover for density-density correlators, by varying the temperature or wave vector, and (iii) logarithmic decay for specific wave vectors of density-density correlators. These anomalous features are observed over time intervals extending up to four decades and strongly resemble predictions of the mode coupling theory (MCT) for state points close to higher-order MCT transitions, which originate from the competition between different mechanisms for dynamic arrest. For the large particles we suggest competition between soft-sphere repulsion and depletion effects induced by neighboring small particles. For the small particles we suggest competition between bulklike dynamics and confinement, respectively induced by neighboring small particles and by the slow matrix of large particles.
\end{abstract}

DOI: 10.1103/PhysRevE.74.021409

PACS number(s): 82.70.Dd, 64.70.Pf, 83.10.Rs

\section{INTRODUCTION}

The rheological properties of soft-matter-based systems can be manipulated by a proper addition of components of different mobilities. Some examples are colloid-polymer mixtures and polymer blends. These types of binary mixtures exhibit unusual relaxation features which challenge the standard pictures of structural dynamic arrest in glass-forming liquids and colloidal systems. Differently from the usual two-step increase and decay for, respectively, mean-squared displacements and dynamic correlators [1-5], the latter do not exhibit a defined plateau at intermediate times between the microscopic and diffusive regimes [6-8]. This result suggests a softer character for the collective caging mechanism-i.e., the temporary trapping of each particle by its neighbors. Dynamic correlators show a highly stretched decay, and for selected values of the control parameters the decay is logarithmic in time. By varying wave vectors or control parameters the decay shows a striking concave-toconvex crossover $[6,8]$, leading to violation of standard scaling laws for complex dynamics as the time-temperature superposition principle.

These anomalous relaxation features have been recently derived within the mode coupling theory (MCT) for simple models of hard-sphere colloids with short-ranged attractions [9]. The MCT is a first-principles theory of the glass transition which makes predictions for averaged dynamic quantities as mean-squared displacements, diffusivities, or incoherent and coherent dynamic correlators $[1,2,10]$. The only input in MCT equations is the structural information contained in the total and partial static structure factors, which enter the memory function accounting for the fluctuating

*Corresponding author. Electronic address: wabmosea@sq.ehu.es forces. MCT determines dynamic quantities from the knowledge of static correlations. Indeed MCT relates small variations in static correlations with large variations in dynamics.

The solution of the MCT equations for the mentioned models of short-ranged attractive colloids $[9,11-14]$ has determined the existence of so-called higher-order transitions, showing properties rather different from the standard fold MCT transition associated with the liquid-glass transition. The mentioned anomalous relaxation features have been related to the presence of nearby higher-order transitions. MCT predictions for these type of models have been confirmed by molecular dynamics simulations $[6,7,15]$ and experiments [16-18].

The models of hard-sphere colloids with short-ranged attractions investigated in Refs. [6,7,9] are used as onecomponent effective models for the colloidal particles in colloid-polymer mixtures. The addition of small polymers (or other small particles) to dense solutions of large colloidal particles yields an effective attraction between the colloidal particles in order to maximize entropy [19]. This effect is known as the depletion mechanism. As a consequence, in a certain range of density, temperature, and mixture composition, competition occurs between two different mechanisms for dynamic arrest of the colloidal particles: hard-sphererepulsion characteristic of colloidal systems and formation of reversible bonds, induced by the depletion mechanism $[20,21]$. In the effective one-component system the higherorder MCT scenario arises as a consequence of these two competing mechanisms of very different localization lengths $[9,13]$. When heating up or cooling down the system, dynamic arrest is exclusively driven by, respectively, hardsphere repulsion and reversible bond formation, and relaxation features of standard liquid-glass transitions are recovered $[6,7]$.

Very recently, we have carried out simulations on a simple bead-spring model for polymer blends [8]. The introduction 
of a significant monomer size disparity yields very different relaxation times for both components in the blend, the component of small monomers being the fast one. The fast component exhibits anomalous relaxation features, very different from standard results observed for homopolymers and strongly resembling predictions of the higher-order MCT scenario for short-ranged attractive colloids. Fully atomistic simulations on a real polymer blend are consistent with the anomalous features reported for the bead-spring model [22]. We have pointed out the hypothesis of an underlying higherorder MCT scenario for the dynamics of the fast component, which might arise from the competition between bulklike caging and confinement as different mechanisms for dynamic arrest for the fast component. Bulklike caging is induced by the particles of the fast component, and confinement is induced by the matrix formed by the chains of the slow component. Due to chain connectivity, the former mechanism is present even for high dilution of the fast component, extending the anomalous relaxation scenario over a broad range of blend compositions [8].

At present no MCT theoretical calculations are available for models of polymer blends similar to that investigated in Ref. [8]. A related system for which theoretical MCT works are available is a binary mixture of large and small hard spheres [23]. However, the size disparity used in such works, and also in computational investigations in the MCT framework [24,25], is not sufficient to provide a large separation in the time scales for large and small particles-i.e., to induce confinement effects for the latter. It is not also sufficient to induce significant depletion effects for the large particles. As a consequence, the anomalous relaxation features reported for polymer blends and short-ranged attractive colloids are not predicted for moderate size disparity and indeed are not observed in the corresponding simulations. Instead, a description in terms of standard MCT predictions is possible for moderate size disparities [23-25].

Very recently, MCT theoretical calculations have been reported by Krakoviack [26,27] for a binary mixture of mobile and static hard spheres, a system where confinement effects are present $[28,29]$. For this system, the extreme cases of the Lorentz gas (a single mobile particle in a disordered medium of static obstacles) and the liquid of hard spheres are obtained for high dilution of, respectively, mobile and static particles. At a given composition of the mixture a higherorder MCT transition has been derived. This result supports the hypothesis of a similar MCT scenario in polymer blends originating from competition between bulklike caging and confinement.

The observed analogies between the dynamics of colloidal particles in colloid-polymer mixtures and of the fast component in polymer blends suggest that the higher-order MCT scenario might be a general feature of systems showing slow dynamics with several competing mechanisms for dynamic arrest. In this article we provide evidence in favor of this hypothesis by carrying out molecular dynamics simulations on a mixture of large and small soft spheres of very different sizes. The results presented here for large size disparity (ratio $\delta=2.5)$ complement previous investigations of slow dynamics in binary mixtures with small disparity. We observe anomalous relaxation features similar to those recently re- ported for models of short-ranged attractive colloids and polymer blends. We investigate a wide range of temperatures and mixture compositions. By tuning the composition, these features are displayed by both the large and small particles.

For the case of the large particles, we assign such anomalous features to competition between soft-sphere repulsion and the depletion mechanism induced by the small particles. For the small particles, we suggest competition between bulklike dynamics induced by the neighboring small particles and confinement induced by the matrix of slow large particles. Similarly to the fast component in polymer blends [8] and despite the absence of chain connectivity, small particles exhibit apparent anomalous relaxation over a broad range of compositions extending up to high dilution. In the latter case, these effects are clearly manifested by a significant subset of small particles forming small clusters.

The article is organized as follows. In Sec. II we introduce the investigated model and give computational details. In Sec. III we present simulation results for static structure factors. We also present dynamic quantities displaying unusual relaxation features. In Sec. IV the framework of the MCT is used in an operational way to describe simulation results. In $\mathrm{Sec}$. V we discuss the possible origin of the observed anomalous dynamic features. Conclusions are given in Sec. VI.

\section{MODEL AND SIMULATION DETAILS}

We have simulated a mixture of large (labeled as $A$ and $B$ ) and small $(C$ and $D)$ particles of equal mass $m=1$, interacting through a soft-sphere potential plus a quadratic term:

$$
V_{\alpha \beta}=4 \epsilon\left[\left(\frac{\sigma_{\alpha \beta}}{r}\right)^{12}-C_{0}+C_{2}\left(\frac{r}{\sigma_{\alpha \beta}}\right)^{2}\right],
$$

where $\epsilon=1$ and $\alpha, \beta \in\{A, B, C, D\}$. The interaction is zero beyond a cutoff distance $c \sigma_{\alpha \beta}$, with $c=1.15$. The addition of the quadratic term to the soft-sphere interaction, with the values $C_{0}=7 c^{-12}$ and $C_{2}=6 c^{-14}$, guarantees continuity of the potential and forces at the cutoff distance. The diameters of the soft-sphere potential for the different types of interaction are $\sigma_{D D}=1, \sigma_{C C}=1.1 \sigma_{D D}, \sigma_{B B}=2.5 \sigma_{D D}, \sigma_{A A}=1.1 \sigma_{B B}$, and $\sigma_{\alpha \beta}=\left(\sigma_{\alpha \alpha}+\sigma_{\beta \beta}\right) / 2$ for the case $\alpha \neq \beta$.

The potential (1) is purely repulsive. It does not show local minima within the interaction range $r<c \sigma_{\alpha \beta}$. Hence, slow dynamics in the present model arises as a consequence of steric effects. MCT theoretical works are usually carried out on systems of hard objects, while simulations in similar systems with continuous interactions are usually preferred for computational simplicity. In the present system, the tail of the interaction potential is progressively probed by decreasing temperature, which plays the role of increasing packing in a system of hard spheres. Hence, the simulations presented here should be useful for a qualitative test of the success or failure of future MCT theoretical works on mixtures of hard spheres with large size disparity.

The composition of the mixture is defined as the fraction of small particles: $x_{\text {small }}=\left(N_{C}+N_{D}\right) /\left(N_{A}+N_{B}+N_{C}+N_{D}\right)$, with $N_{\alpha}$ denoting the number of particles of the species $\alpha$. As shown below, the introduction of a large size disparity be- 
tween the sets $\{A, B\}$ and $\{C, D\}$ yields very different time scales for both sets. We impose the constraints $N_{A}=N_{B}$ and $N_{C}=N_{D}$. These constraints, together with the small selected ratios $\sigma_{C C} / \sigma_{D D}=\sigma_{A A} / \sigma_{B B}=1.1$, guarantee that only very small dynamic differences are induced between particles within a same set $(\{A, B\}$ or $\{C, D\})$ and, at the same time, avoid crystallization for the investigated compositions $x_{\text {small }}$. Crystallization would occur for very asymmetric mixtures if only one type of large and small particles were introduced [30].

In the following, temperature $T$, distance, wave vector $q$, and time $t$ will be given, respectively, in units of $\epsilon / k_{B}, \sigma_{D D}$, $\sigma_{D D}^{-1}$, and $\sigma_{D D}(m / \epsilon)^{1 / 2}$. The packing fraction $\phi$ is defined as

$$
\phi=\frac{\pi}{6 L^{3}}\left[N_{A} \sigma_{A A}^{3}+N_{B} \sigma_{B B}^{3}+N_{C} \sigma_{C C}^{3}+N_{D} \sigma_{D D}^{3}\right],
$$

with $L$ the side of the simulation box. Simulations have been carried out at a constant packing fraction $\phi=0.53$. This value is comparable to those used in simulations of slow relaxation in simple liquids. For comparison, the original LennardJones binary mixture investigated by Kob and Andersen [3] has $\phi=0.59$ with the definition of packing fraction given above. We investigate the $T$ dependence of the dynamics for mixture compositions $x_{\text {small }}=0.1,0.3,0.6$, and 0.8 . The numbers of large and small particles for each composition are, respectively, $\left(N_{A}+N_{B}: N_{C}+N_{D}\right)=(5400: 600), \quad(2100: 900)$, (1000:1500), and (800:3200). The system is prepared by placing the particles randomly in the simulation box, with a constraint that avoids core overlapping. Periodic boundary conditions are implemented. Equations of motion are integrated by using the velocity Verlet scheme [31], with a time step ranging from $2 \times 10^{-4}$ to $5 \times 10^{-3}$, for, respectively, the highest and lowest investigated temperature. A link-cell method [31] is used for saving computational time in the determination of particles within the cutoff distance of a given one.

At each state point, the system is thermalized at the requested temperature by periodic velocity rescaling. After reaching equilibrium, energy and pressure show no drift. Likewise, mean-squared displacements and dynamic correlators show no aging-i.e., no time shift when being evaluated for progressively longer time origins. Once the system is equilibrated, a microcanonical run is performed for the production of configurations, from which static structure factors, mean-squared displacements, and dynamic correlators are computed. For each state point, the latter quantities are averaged over typically $20-40$ independent samples.

\section{RESULTS}

\section{A. Static structure factors}

We compute normalized partial static structure factors $S_{\alpha \beta}(q)=\left\langle\rho_{\alpha}(q, 0) \rho_{\beta}(-q, 0)\right\rangle / \sqrt{N_{\alpha} N_{\beta}}, \quad$ with $\quad \rho_{\alpha}(q, t)$ $=\Sigma_{j} \exp \left[i \mathbf{q} \cdot \mathbf{r}_{\alpha, j}(t)\right]$, the sum extending over all the particles of the species $\alpha \in\{A, B, C, D\}$. Figure 1 shows, at a fixed temperature $T=0.50$ and different mixture compositions, results for $A-A, D-D$, and $A-D$ pairs. Data for other large-large, small-small, and large-small pairs display only small quantitative differences.
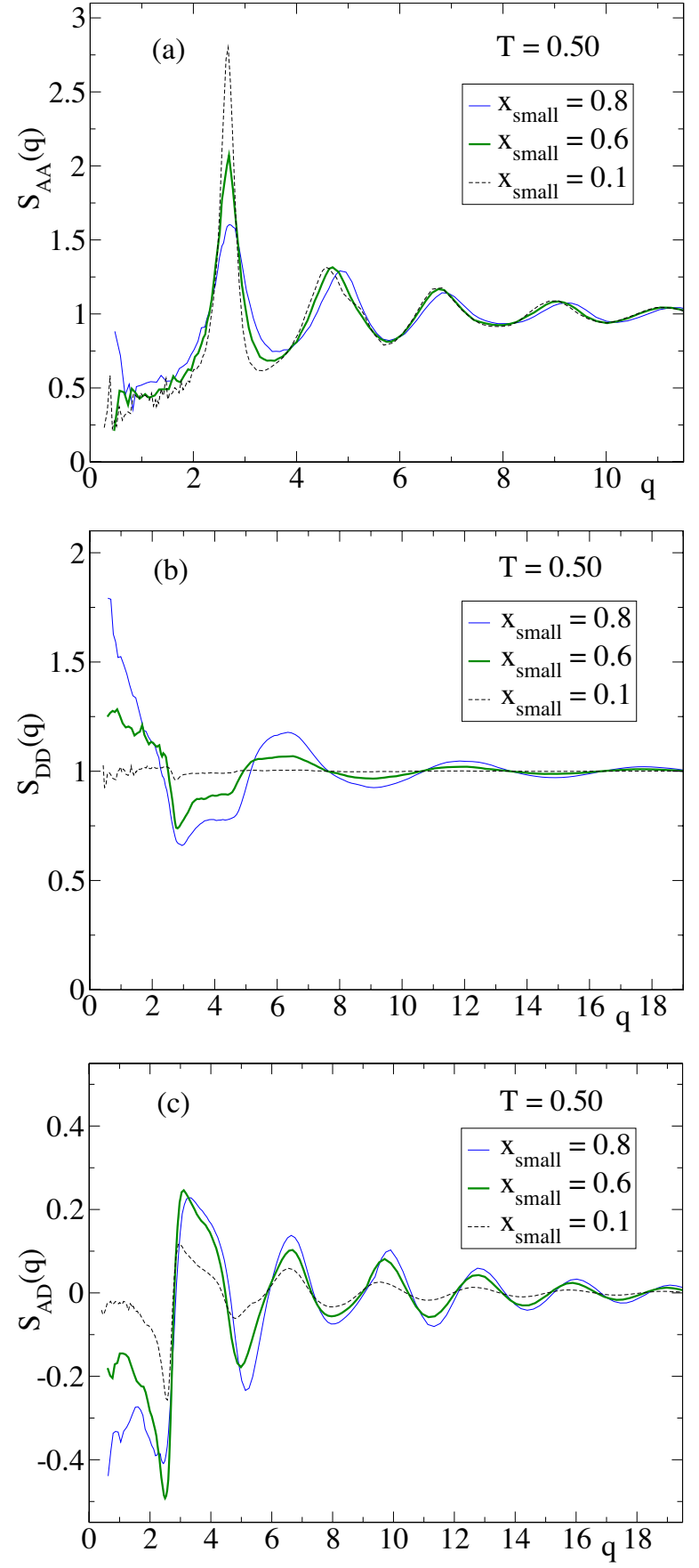

FIG. 1. (Color online) Partial static structure factors at $T=0.50$ and different mixture compositions, as computed from simulation data. Wave vectors are given in units of $\sigma_{D D}^{-1}$.

For high concentration of the large particles, $x_{\text {small }}=0.1$, $S_{\mathrm{AA}}(q)$ shows a sharp first peak at $q=2.65 \sigma_{D D}^{-1}$, corresponding to a typical distance of $2.37 \sigma_{D D}$ between neighboring $A$ particles. This distance is smaller than the soft-sphere diameter $\sigma_{A A}=2.75 \sigma_{D D}$. This feature is possible due to the interpenetrable character of soft spheres. By increasing the concentration of small particles, the matrix of large particles progressively becomes more disordered. As a consequence, the height of the first peak of $S_{A A}(q)$ decreases considerably. The first minimum follows the opposite trend and becomes 
less pronounced. The small shift of maxima and minima to higher $q$ values by increasing $x_{\text {small }}$ reflects a stronger packing of the large particles. For large concentration of small particles, $x_{\text {small }}=0.8, S_{A A}(q)$ shows a small peak at low $q$. We assign this peak to the presence of inhomogeneities or "voids" in the matrix of large particles, which are filled by the small particles [see also Sec. V, Fig. 11(c)].

At high dilution of the small particles, $x_{\text {small }}=0.1, S_{D D}(q)$ shows a nearly structureless profile, close to the flat behavior expected for a gas. Only a weak oscillation is observed at low $q$. This feature reflects the existence of a small fraction of clusters of neighboring small particles (see also Sec. V). Increasing the concentration of small particles yields a broad peak at $q \approx 6.3 \sigma_{D D}^{-1}$, corresponding to a typical distance of about $\sigma_{\mathrm{DD}}$ between neighboring small particles. The peak grows up and narrows by increasing $x_{\text {small }}$, as a signature of progressive ordering of the small particles. Still, one finds a rather broad peak at the highest value of $x_{\text {small }}=0.8$. Increasing the concentration of small particles also produces a low$q$ peak of increasing intensity in $S_{D D}(q)$. Such a peak originates from the inhomogeneities in the structure formed by the small particles [see also Sec. V, Figs. 11(b) and 11(c)]. We do not observe changes, at any of the investigated temperatures, in the intensity of the low- $q$ peak within the time window of the simulation. Hence, phase separation is discarded for the results here presented.

The static structure factor for cross correlations, $S_{A D}(q)$, exhibits a first peak at $q \approx 3.2 \sigma_{D D}^{-1}$, corresponding to a typical distance of about $2 \sigma_{D D}$ between neighboring large and small particles (for comparison the soft-sphere diameter for $A-D$ interactions is $\left.\sigma_{A D}=1.875 \sigma_{D D}\right)$. The intensity at large wavelength (low $q$ ) becomes more negative by increasing the concentration of small particles. This effect might originate from nonrandom mixing of large and small particles [see also Sec. V, Figs. 11(b) and 11(c)]. Only for high dilution of the small particles is mixing approximately random and the limit $S_{A D}(q \rightarrow 0)=0$ is approached.

\section{B. Diffusivities and mean-squared displacements}

Figure 2 shows the $T$ dependence of the diffusivity $D$ for all the species in the mixture at all the investigated compositions. For each species $\alpha \in\{A, B, C, D\}$ the diffusivity is calculated from the long-time limit of $\left\langle\left[\Delta r_{\alpha}(t)\right]^{2}\right\rangle / 6 t$, with $\left\langle\left[\Delta r_{\alpha}(t)\right]^{2}\right\rangle$ the corresponding mean-squared displacement at time $t$. As shown in Fig. 2, the introduction of a significant size disparity yields very different time scales for the sets of large and small particles. Small differences are instead obtained between the diffusivities of both species within a same set. Only for the lowest temperature at concentration $x_{\text {small }}$ $=0.1$ there are significant differences (about a factor of 4 in diffusivity) between $C$ and $D$ particles. In the following, simulation results will only be shown for the large $A$ particles and the small $D$ ones. The qualitive behavior of respectively $B$ and $C$ particles is the same, displaying only small quantitative differences with the formers.

Figure 3 shows the $T$ dependence of the mean-squared displacement of the $A$ particles, $\left\langle\left(\Delta r_{A}\right)^{2}\right\rangle$, for three different compositions $x_{\text {small }}=0.1,0.6$, and 0.8 . As usually observed in

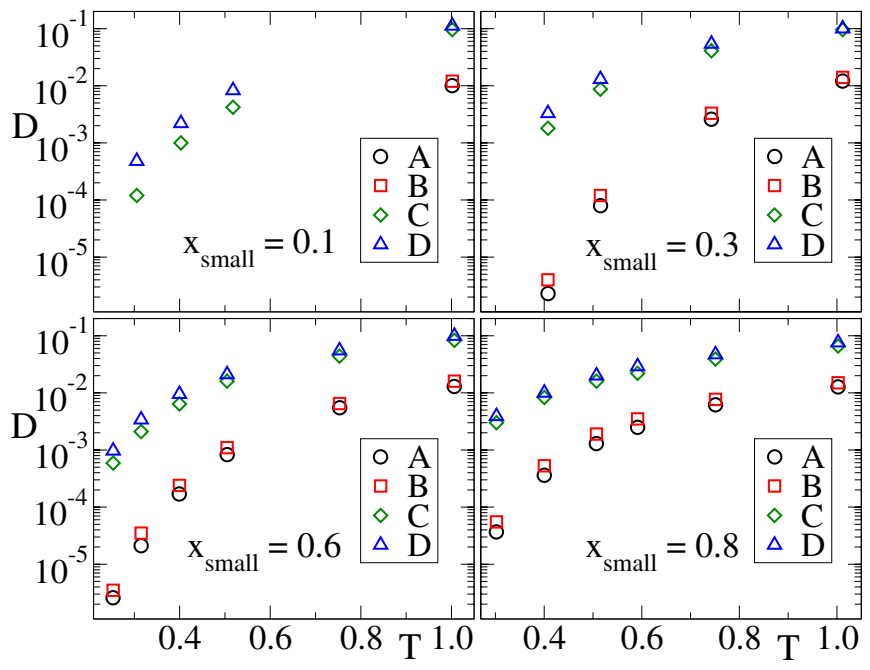

FIG. 2. (Color online) For the investigated compositions, the temperature dependence of the diffusivities for the different species of the mixture. For low temperatures at $x_{\text {small }}=0.1$, data for the large particles are absent, since they do not reach the diffusive regime in the time window of the simulation [see text and Fig. 3(a)].

the proximity of liquid-glass transitions [2-4], a bending occurs after the initial ballistic $\left(\propto t^{2}\right)$ regime. A plateau arises at low temperatures. This effect corresponds to the well-known caging regime-i.e., the temporary trapping of each particle in the cage formed by the neighboring ones. At long times, the diffusive regime $(\propto t)$ is reached for values $\left\langle\left(\Delta r_{A}\right)^{2}\right\rangle$ $\lesssim \sigma_{A A}^{2}$-i.e, when the $A$ particles have moved, on average, a distance of the order of their size.

By looking in more detail at the data for $T=0.75$ and $T$ $=1.0$, at $x_{\text {small }} \geq 0.6$ [Figs. 3(b) and 3(c)], an unusual approximate sublinear behavior $\propto t^{\alpha}$ is observed over two time decades after the ballistic regime. The exponent $\alpha<1$ decreases by decreasing temperature. For $x_{\text {small }}=0.8$ we also note, at all temperatures, a bump at the interval $0.2 \lesssim t$ $\leq 0.4$.

Figure 4 shows, at the same compositions, results for $D$ particles. Remarkable differences with the mean-squared displacements of $A$ particles are observed. Differently from $A$ particles (and from the standard behavior in the proximity of liquid-glass transitions) $D$ particles reach the diffusive regime $\propto t$ for displacements much larger than their size: from $\left\langle\left(\Delta r_{D}\right)^{2}\right\rangle \sim 10 \sigma_{D D}^{2}$ at high $T$ to $\left\langle\left(\Delta r_{D}\right)^{2}\right\rangle \sim 100 \sigma_{D D}^{2}$ at low $T$. This result is observed for all the compositions. As in the case of the $A$ particles, for $x_{\text {small }} \geq 0.6$ an unusual approximate sublinear regime is observed at intermediate times, with an exponent decreasing by decreasing temperature. This sublinear regime sets on for $\left\langle\left(\Delta r_{D}\right)^{2}\right\rangle \gtrsim \sigma_{D D}^{2}$.

The results reported in this subsection evidence the existence of unusual relaxation features in the slow dynamics of mixtures of large and small particles with sufficiently large size disparity. Next we evaluate the effects of size disparity in partial density-density correlators.

\section{Density-density correlators}

We compute partial density-density correlators $F_{\alpha \beta}(q, t)$ $=\left\langle\rho_{\alpha}(q, t) \rho_{\beta}(-q, 0)\right\rangle /\left\langle\rho_{\alpha}(q, 0) \rho_{\beta}(-q, 0)\right\rangle$. Figure 5 shows the 

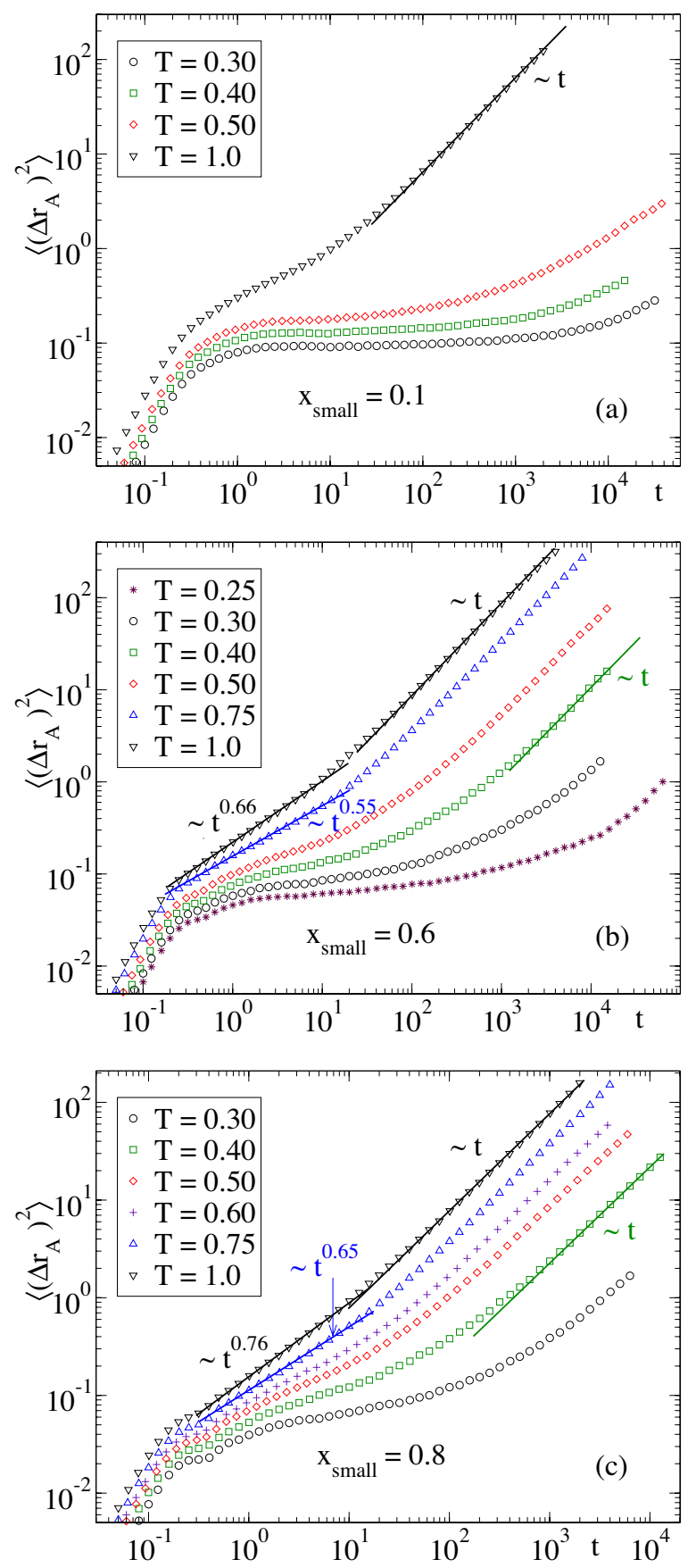

FIG. 3. (Color online) Symbols: mean-squared displacement of $A$ particles for different compositions of the mixture. Straight lines indicate linear or sublinear behavior.

$T$ dependence of $F_{A A}(q, t)$ at specific values of the wave vector $q$ and different compositions. For the case $x_{\text {small }}=0.1$ [Fig. 5(a)], A-A correlations display the standard behavior observed for liquid-glass transitions [1-5]. After the initial transient regime, $F_{A A}(q, t)$ shows a first decay to a plateau. By decreasing the temperature, the plateau extends over longer time intervals. At long times, a second decay occurs from the plateau to zero. This second decay corresponds to the $\alpha$ process of the glass transition and is usually well described by a stretched exponential function.
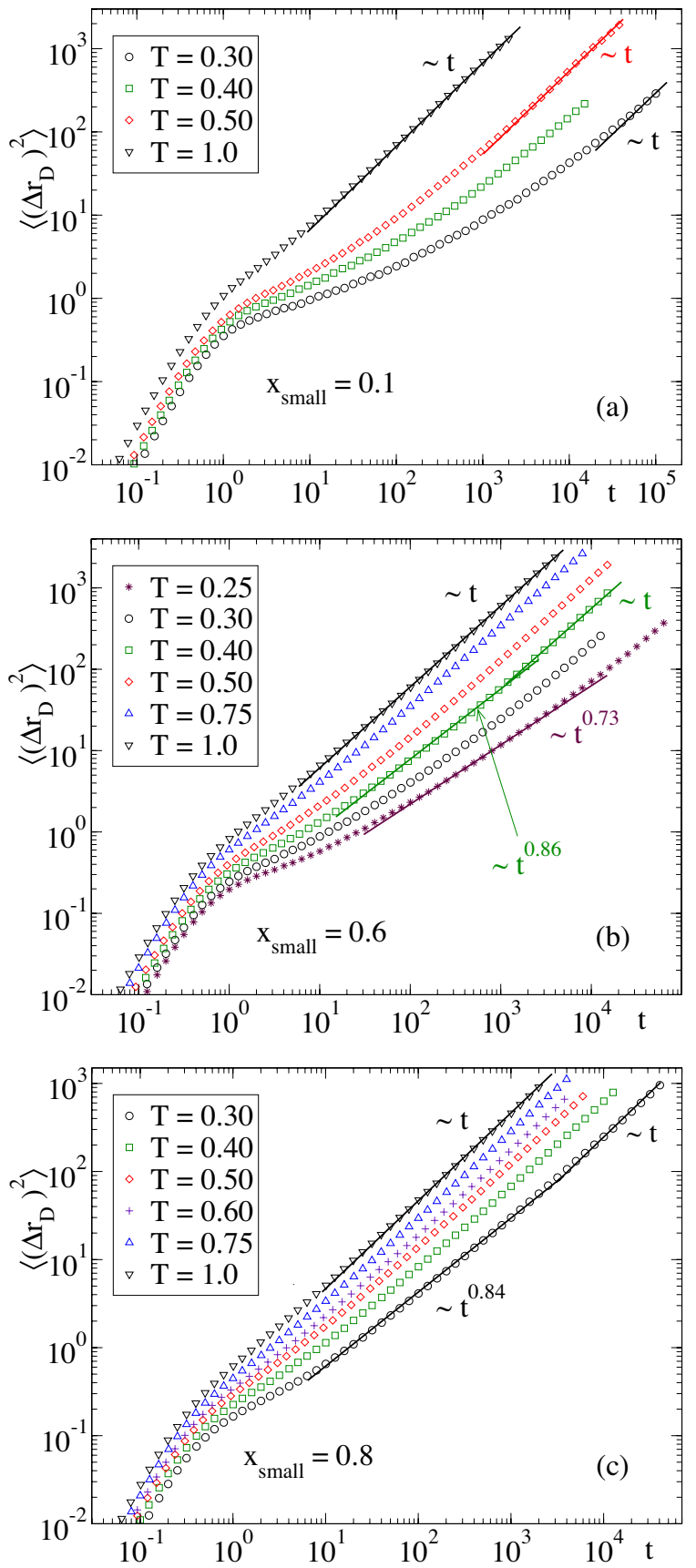

FIG. 4. (Color online) As in Fig. 3 for $D$ particles.

Figures 5(b) and 5(c) show results for the case $x_{\text {small }}$ $=0.8$, for wavelengths $(2 \pi / q)$ of, respectively, $2.2 \sigma_{D D}$ and $1.1 \sigma_{D D}$. While apparently standard behavior is obtained in the former case, unusual features are observed for wavelengths probing the size of the small particles [Fig. 5(c)]. First, $F_{A A}(q, t)$ does not exhibit a defined plateau. Moreover, the shape of the long-time decay shows a concave-to-convex crossover by decreasing temperature. At an intermediate temperature $T=0.50$ the decay is logarithmic over two time decades. As in the case of the mean-squared displacement, a bump is observed at the interval $0.2 \lesssim t \leqslant 0.4$.

Unusual relaxation features are also displayed by $F_{D D}(q, t)$. Figure 6 shows results for different compositions 

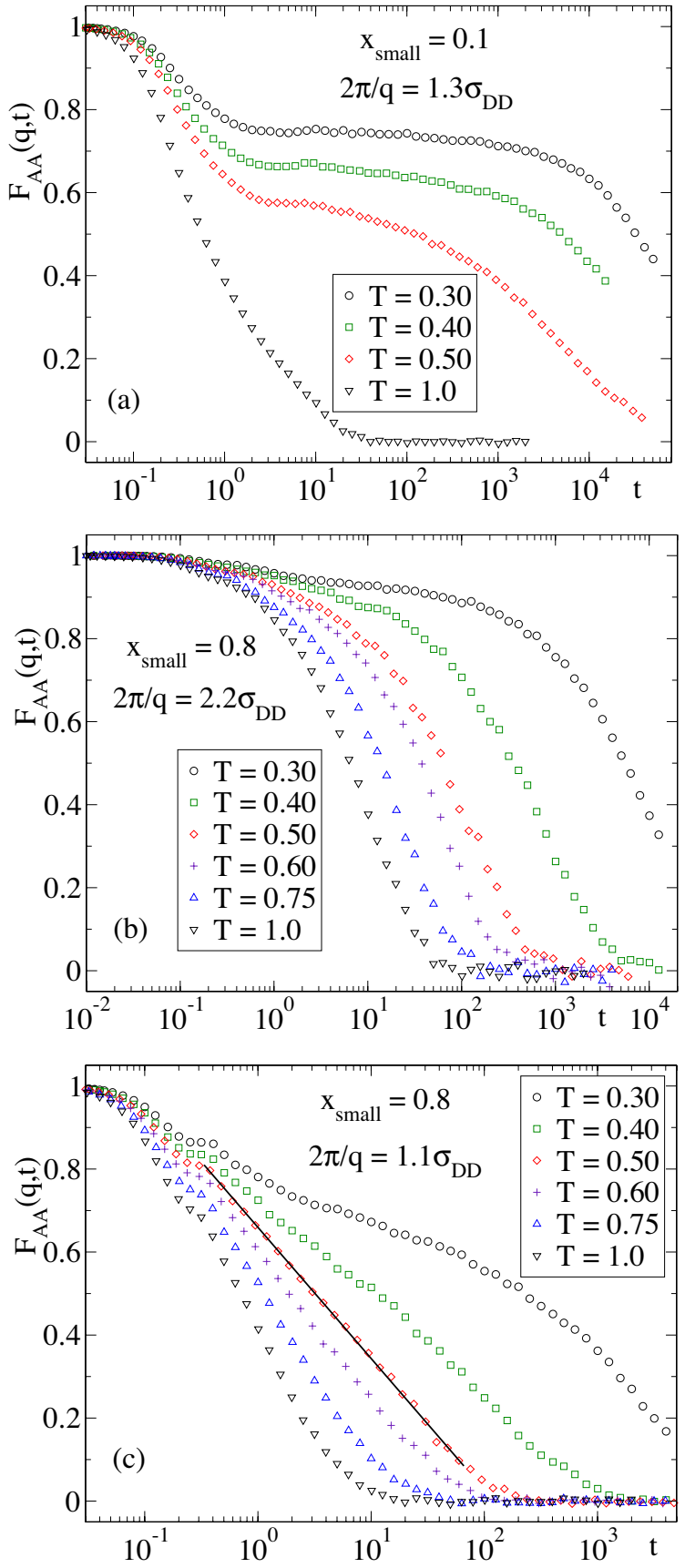

FIG. 5. (Color online) Symbols: $T$ dependence of $F_{A A}(q, t)$, at specific $q$ values, for several compositions. The straight line in panel (c) indicates logarithmic decay.

at wavelengths probing the size of the large particles. No defined plateaus are observed for $x_{\text {small }}=0.1$ and 0.6. Logarithmic relaxation is observed at intermediate temperatures for time intervals extending up to three decades. Data for $x_{\text {small }}=0.6$ exhibit a concave-to-convex crossover. Due to the mentioned partial crystallization of large particles for $x_{\text {small }}$ $=0.1$ [30], data are absent in Fig. 6(a) for the range 0.50 $<T<1.0$. Apparently, a concave-to-convex crossover is also present for this composition, though data are not conclusive.

The results for $F_{D D}(q, t)$ at $x_{\text {small }}=0.8$ display a qualitatively different behavior. At the lowest investigated tempera-
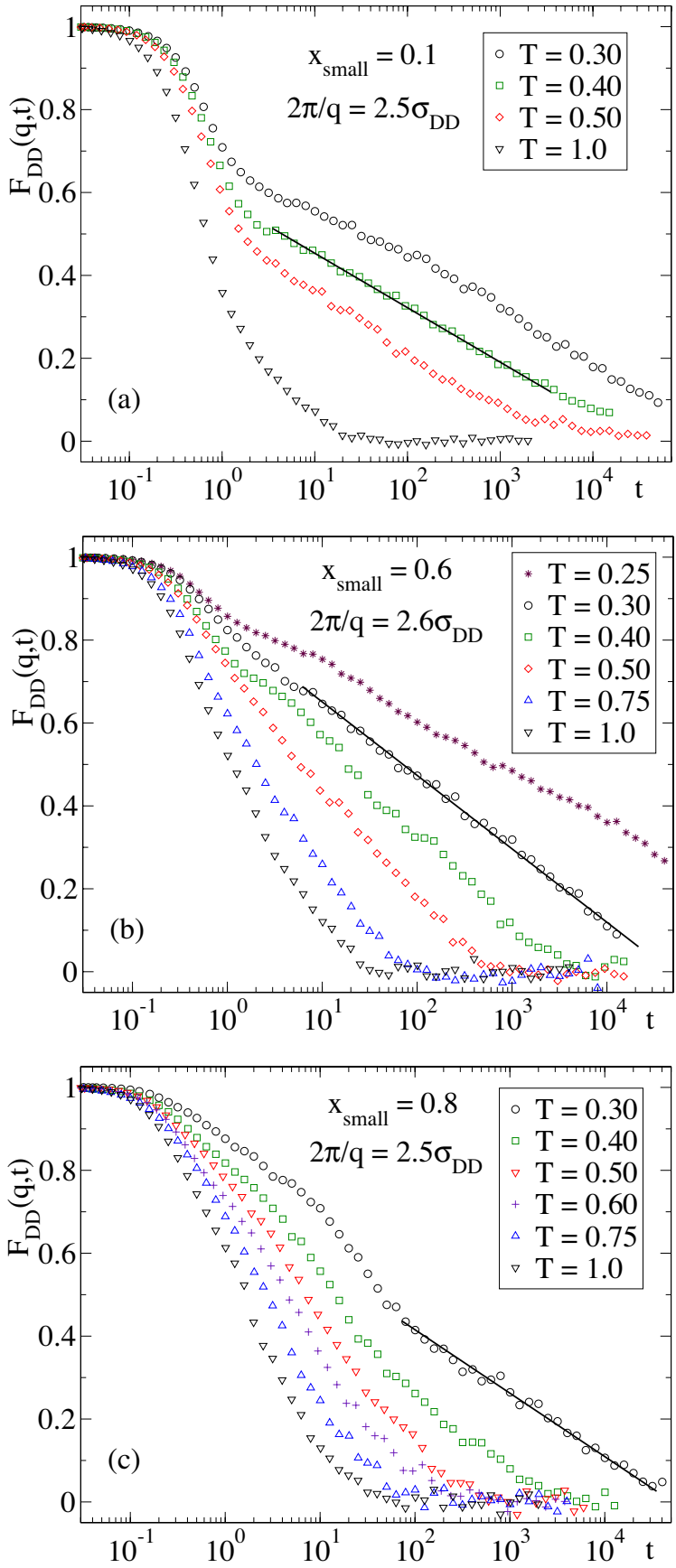

FIG. 6. (Color online) As in Fig. 5 for $F_{D D}(q, t)$.

ture $T=0.30$ a bump is observed at the interval $3<t<6$, followed by a decay until $t \approx 60$, where logarithmic relaxation sets on and extends over three time decades.

\section{MCT ANALYSIS}

\section{A. Large particles}

Many of the anomalous relaxation features reported in Sec. III-sublinear behavior for mean-squared displacements and logarithmic decay and concave-to-convex crossover for density-density correlators-strongly resemble those reported for hard-sphere colloids with short-ranged attractions 
$[6,7]$ and for the fast component in polymer blends with components of very different mobilities [8,22]. As mentioned in the Introduction, these anomalous features have been strictly derived in the framework of the MCT for simple models of short-ranged attractive colloids [9]. According to $\mathrm{MCT}$, this anomalous relaxation scenario arises from an underlying higher-order transition. Motivated by this fact, we discuss the present results by using MCT in an operational way.

In its ideal version, which neglects activated hopping events, MCT predicts a sharp transition from an ergodic liquid to a nonergodic arrested state at a given value $x_{\mathrm{c}}$ of the relevant control parameter $x$ (in practice density or temperature) $[1,2,10]$. When crossing the transition point $x=x_{\mathrm{c}}$ from the ergodic to the arrested state, the long-time limit of the density-density correlator for wave vector $q, F(q, t)$, jumps from zero to a nonzero value, denoted as the critical nonergodicity parameter $f_{q}^{\mathrm{c}}$. Moving beyond the transition point into the nonergodic state yields a progressive increase of the nonergodicity parameter, $f_{q}>f_{q}^{\mathrm{c}}$. In the MCT formalism, the standard liquid-glass transition is of the fold type, also denoted as $A_{2}[1,2,10,32]$. In the standard case the jump in $F(q, t)$ is discontinuous; i.e., the critical nonergodicity parameter $f_{q}^{\mathrm{c}}$ takes a finite value. MCT transitions with $f_{q}^{\mathrm{c}}>0$ are also denoted as type- $B$ transitions. For ergodic states close to the transition point, the initial part of the $\alpha$ process-i.e., the von Schweidler regime-is approximated by a power law expansion[1,2,10]

$$
F(q, t) \approx f_{q}^{\mathrm{c}}-h_{q}(t / \tau)^{b}+h_{q}^{(2)}(t / \tau)^{2 b},
$$

with $0<b \leq 1$. The prefactors $h_{q}$ and $h_{q}^{(2)}$ only depend on the wave vector $q$. The characteristic time $\tau$ only depends on the separation parameter $\left|x-x_{\mathrm{c}}\right|$ and is divergent at the transition point. Another important prediction of the MCT for state points close to fold transitions is the so-called second universality or $x$-time superposition principle (with $x$ the corresponding control parameter). According to this prediction, the final decay of $F(q, t)$ (i.e., the final part of the $\alpha$ process) is invariant under scaling by the $\alpha$-relaxation time $\tau_{\alpha}$. Hence, for two state points $x=x_{1}$ and $x=x_{2}$ close to the transition point the final decay of $F(q, t)$ fulfills the relation $[1,2,10]$

$$
F_{x=x_{1}}\left(q, \frac{t}{\tau_{\alpha}\left(x_{1}\right)}\right)=F_{x=x_{2}}\left(q, \frac{t}{\tau_{\alpha}\left(x_{2}\right)}\right) .
$$

The $\alpha$-relaxation time $\tau_{\alpha}$ is a time scale probing the $\alpha$ process. In practice, it can be obtained from fitting the decay from the plateau to a stretched exponential $A_{q} \exp \left[-\left(t / \tau_{\alpha}\right)^{\beta}\right]$, with $A_{q}$ the plateau height and $0<\beta<1$. It can also be defined as the time where $F(q, t)$ decays to some small valuee.g., $0.3\left(\tau_{0.3}\right)$-provided it is well below the plateau.

Another prediction of the MCT for points close to an $A_{2}$ transition is the power-law decrease of the diffusivity to zero, $D \propto\left|x-x_{\mathrm{c}}\right|^{\gamma}$. The exponent $\gamma$ is given by $[1,2,10]$

$$
\gamma=\frac{1}{2 a}+\frac{1}{2 b}
$$

with $0<a<0.4$. Hence $\gamma \geq 1.75$. The critical exponents $a, b$, and $\gamma$ are related to the so-called exponent parameter $1 / 2$ $\leq \lambda \leq 1$ through the relation

$$
\lambda=\frac{\Gamma^{2}(1+b)}{\Gamma(1+2 b)}=\frac{\Gamma^{2}(1-a)}{\Gamma(1-2 a)},
$$

with $\Gamma$ the gamma function $[1,2,10]$.

By numerically solving the MCT equations for dynamic correlators, transition points are determined as those where the respective long-time limit exhibits a jump from a zero to a nonzero value. From the knowledge of the total and partial static correlations at the transition point, all the critical exponents and the coefficients in Eqs. (3), (5), and (6) are univoquely determined $[1,2,10]$. Solving the MCT equations is a difficult task, which in general is only possible for rather simplified models of real systems. Hence, instead of solving the equations, critical exponents and prefactors are often obtained as fit parameters from simulations or experimental data. Consistency of the data analysis requires that the soobtained critical exponents fulfill Eqs. (5) and (6).

Figure 7 shows an analysis of data for $A$ particles at composition $x_{\text {small }}=0.6$ in the framework of the MCT for $A_{2}$ transitions. Figure 7(a) shows a fit of the diffusivity to a power law $D_{A} \propto\left(T-T_{\mathrm{c}}\right)^{\gamma}$. The fit provides the values $T_{\mathrm{c}}=0.180$ and $\gamma=3.80$. From Eqs. (5) and (6) we obtain $\lambda=0.887, a$ $=0.220$, and $b=0.326$. Figure $7(\mathrm{~b})$ shows, for $F_{A A}(q, t)$ at a low $T$, fits of the decay from the plateau to Eq. (3). By forcing the von Schweidler exponent to the mentioned value $b=0.326$ a consistent description is achieved. Figure 7(c) shows a successful test of the second universality (timetemperature superposition principle), by using $\tau_{0.3}$ for the $\alpha$-relaxation time. For the composition $x_{\text {small }}=0.3$ similar results are obtained (not shown). From an analogous analysis we obtain $T_{\mathrm{c}}=0.344, \lambda=0.880, \gamma=3.68, a=0.226$, and $b$ $=0.340$ for this latter composition.

For the composition $x_{\text {small }}=0.1$, a test of MCT for $A$ particles is not possible due to the absence of data in the range $0.50<T<1.0$. Though a two-step decay is observed for $T$ $\leq 0.50$ [see Fig. 5(a)], a fit to Eq. (3) is not possible in that temperature range, since the plateau height is not constant, but clearly increases by decreasing temperature. According to predictions of ideal MCT, these temperatures would correspond to nonergodic states below the transition point $\left(T<T_{\mathrm{c}}\right)$. Within this interpretation, the fact that a decay from the plateau occurs at long times would be a signature of the so-called hopping events, which restore ergodicity.

For the composition $x_{\text {small }}=0.8$, data of $F_{A A}(q, t)$ for wavelengths $2 \pi / q$ probing the size of the small particles do not show a defined plateau [Fig. 5(c)], and a fit to Eq. (3) is not possible. Moreover, the observed logarithmic decay and concave-to-convex crossover do not fit to expectations for $A_{2}$ transitions. They instead resemble the features reported for short-ranged attractive colloids [6,7] and polymer blends [8], which for the former case have been derived in the frame- 

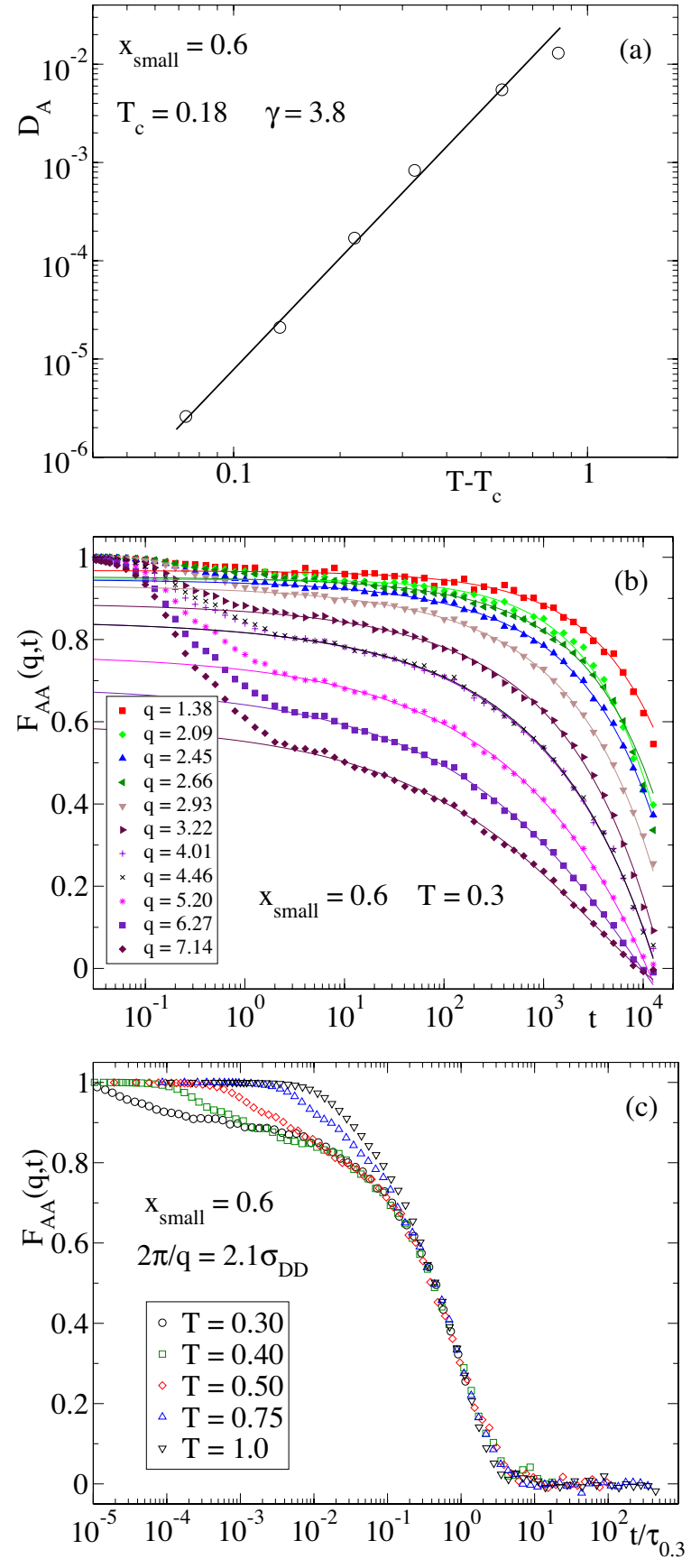

FIG. 7. (Color online) MCT analysis of data for $A$ particles, for composition $x_{\text {small }}=0.6$. Panel (a): $T$ dependence of the diffusivity. Symbols are simulation data. The line is a fit to a MCT power law $D_{A} \propto\left(T-T_{\mathrm{c}}\right)^{\gamma}$ (see text). Panel (b): (symbols) $q$ dependence of $F_{A A}(q, t)$ at a low temperature. Curves are fits to Eq. (3). Panel (c): $T$ dependence of $F_{A A}(q, t)$ for a specific $q$ value. In order to test the second universality, times are divided by the $\alpha$-relaxation time $\tau_{0.3}(T)$ (see text).

work of MCT for state points close to higher-order transitions.

A higher-order MCT transition $A_{n+1}$ is characterized by an exponent parameter $\lambda=1$ and can emerge as the result from the interplay between $n$ control parameters $\left\{\mathbf{x}^{n}\right\}$ $=\left(x_{1}, x_{2}, \ldots, x_{n}\right)$. Higher-order MCT transitions were initially derived for schematic models $[33,34]$, but only recently have been obtained for short-ranged attractive colloids [9,11-14] as a first realization in real systems. It can be demonstrated that an expansion in a power-law series as Eq. (3) is not convergent for $\lambda=1$ or for values of $\lambda$ very close to unity. On the contrary, a logarithmic expansion is rapidly convergent. Hence, close to a higher-order MCT transition, or more generally to an $A_{2}$ transition with $\lambda \lesssim 1, F(q, t)$ is approximated at intermediate times as [9,33-35]

$$
F(q, t) \approx f_{q}^{c}-H_{q} \ln (t / \tau)+H_{q}^{(2)} \ln ^{2}(t / \tau),
$$

where the prefactors $H_{q}$ and $H_{q}^{(2)}$ depend on $q$ and on the distance of the state point $\left\{\mathbf{x}^{n}\right\}$ to the transition point $\left\{\mathbf{x}_{c}^{n}\right\}$. These prefactors exhibit two important properties [9]: (i) $H_{q}$ factorizes as $H_{q}=C\left(\left\{\mathbf{x}^{n}\right\}\right) \tilde{H}_{q}$ where $\tilde{H}_{q}$ only depends on $q$ and the $q$-independent term $C\left(\left\{\mathbf{x}^{n}\right\}\right)$ depends on the state point. Hence, values of $H_{q}$ for different state points close to the transition point must be proportional. (ii) $H_{q}^{(2)}$ does not follow scaling behavior. It is decomposed as [9]

$$
H_{q}^{(2)}=A\left(\left\{\mathbf{x}^{n}\right\}\right)+B\left(\left\{\mathbf{x}^{n}\right\}\right) K_{q},
$$

where $K_{q}$ only depends on $q$ and the $q$-independent terms $A\left(\left\{\mathbf{x}^{n}\right\}\right)$ and $B\left(\left\{\mathbf{x}^{n}\right\}\right)$ depend on the state point. The terms $\widetilde{H}_{q}$ and $K_{q}$ are univoquely determined by static structure factors at the higher-order transition point. As in the case of $A_{2}$ transitions, the coefficients in Eq. (7) are often obtained as fit parameters from simulations or experimental data.

Decomposition of $H_{q}^{(2)}$ according to Eq. (8) has an important consequence. There are hypersurfaces in the control parameter space, $\left\{\mathbf{x}^{n}\right\}=\left\{\mathbf{x}^{n}\right\}(q)$, where $H_{q}^{(2)}$ changes its sign, being zero along the hypersurface. This property leads, for a given value of $q$, to a concave-to-convex crossover in $F(q, t)$ when crossing the hypersurface by varying control parameters (as temperature or density) [9]. Analogously, for a given state point, varying the value of $q$ also leads to a concave-to-convex crossover in $F(q, t)$. Since $H_{q}^{(2)}=0$ for state points at the hypersurface, according to Eq. (7), $F(q, t)$ will exhibit a logarithmic decay for such state points. Moving between different state points changes the value of $q$ for which pure logarithmic decay occurs. The concave-toconvex crossover is one of the main signatures of the higherorder MCT scenario and differentiates it from other theoretical frameworks [9].

As shown in Figs. 5(c) and 8(a), a concave-to-convex crossover is present, by both varying the temperature and wave vector, for $F_{A A}(q, t)$ at composition $x_{\text {small }}=0.8$. Figure 8(a) shows fits to Eq. (7) for $T=0.50$ [36]. Figures 8(b) and 8(c) show, respectively, the obtained values for $f_{q}^{\mathrm{c}}$ and $H_{q}$, for temperatures $T=0.40$ and $T=0.50$. The fact that a common $f_{q}^{\mathrm{c}}$ is found for both states, together with the observed scaling behavior of $H_{q}$, would be consistent with the existence of a nearby MCT higher-order transition $(\lambda=1)$ or an $A_{2}$ transition with $\lambda \lesssim 1$. Differently from the usual behavior for liquid-glass transitions, $f_{q}^{\mathrm{c}}$ shows no strong modulation but a nearly monotonous decay. Though this result qualitatively resembles the observed $q$ dependence at $A_{3}$ and $A_{4}$ transitions for models of short-ranged attractive colloids 

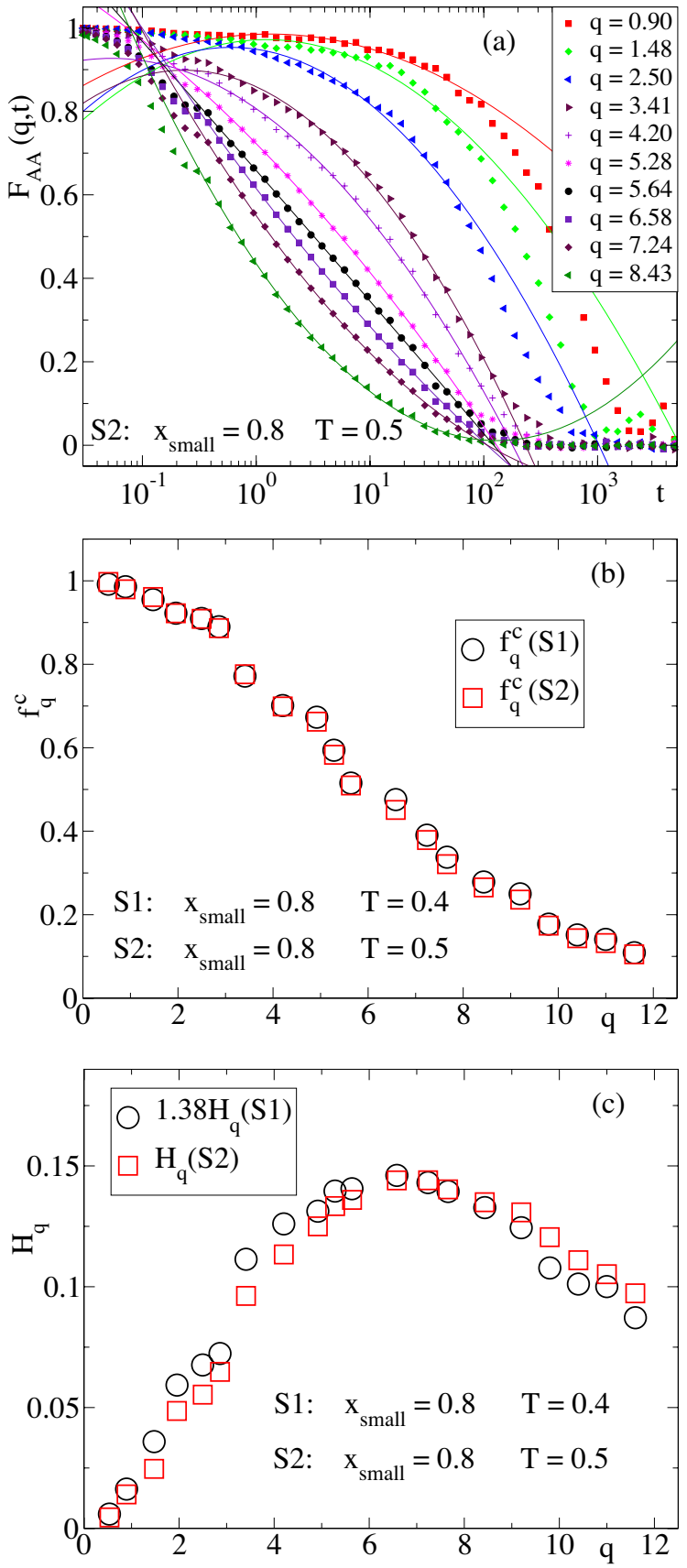

FIG. 8. (Color online) Panel (a): (symbols) $q$ dependence of $F_{A A}(q, t)$ for a composition $x_{\text {small }}=0.8$ at a low temperature. Curves are fits to Eq. (7). Panels (b) and (c): values of, respectively, $f_{q}^{\mathrm{c}}$ and $H_{q}$, as obtained from fits to Eq. (7) of $F_{A A}(q, t)$ at two state points $S 1$ and $S 2$. The characteristic times $\tau$ for $S 1$ and $S 2$ are, respectively, 9 and 3 .

$[6,9,13,14,37]$, such a comparison can be misleading. The observed $q$ dependence is not necessarily related to a hypothetical higher-order MCT transition. It is indeed also found at moderate size disparity, for which standard relaxation features are observed $[23,25]$.

It is also noteworthy that the observed sublinear behavior in the mean-squared displacement at intermediate times is another feature characteristic of the higher-order MCT scenario. In leading-order [9], the mean-squared displacement is given by $\left\langle[\Delta r(t)]^{2}\right\rangle=r_{\mathrm{c}}^{2}(t / \tau)^{z}$, with $r_{\mathrm{c}}$ the localization length and $z=h_{\mathrm{MSD}} C\left(\left\{\mathbf{x}^{n}\right\}\right) / r_{\mathrm{c}}^{2}$. The coefficient $h_{\mathrm{MSD}}$ is determined by static structure factors at the transition point. Since the prefactor $C\left(\left\{\mathbf{x}^{n}\right\}\right)$ decreases as the transition point is approached, the exponent $z$ also decreases, as observed in Fig. 3(c). Deviations from pure power-law behavior result from corrections to the leading term. Such corrections are minimal for certain values of the control parameters, yielding pure power-law behavior [9].

\section{B. Small particles}

Data for $D$ particles cannot be reproduced by the standard $A_{2}$ scenario for any of the investigated compositions, due to the absence of defined plateaus and to the presence of logarithmic relaxation in density-density correlators. Data for $x_{\text {small }}=0.6$ exhibit features resembling predictions of the higher-order MCT scenario. Figure 9 shows results from such an analysis, analogous to that in Fig. 8 for $A$ particles, in terms of the latter framework. The concave-to-convex crossover in $F_{D D}(q, t)$, obtained by decreasing temperature at constant $q$ [Fig. 6(b)], is also observed by varying $q$ at constant temperature [Fig. 9(a)]. A good description of the decay is achieved by Eq. (7). Consistently, fits for different state points provide the same values for $f_{q}^{\text {c }}$ [Fig. 9(b)] and scaling behavior is obtained for the corresponding values of $H_{q}$ [Fig. 9(c)]. The observed sublinear behavior for the mean-squared displacement and the decrease of the exponent by decreasing temperature [Fig. 4(b)] are other features shared with the higher-order MCT scenario.

We have performed an analogous analysis for $F_{D D}(q, t)$ at composition $x_{\text {small }}=0.1$ (see Fig. 10), despite there being some differences with features characteristic of the higherorder MCT scenario. Differently from the case $x_{\text {small }}=0.6$, no apparent sublinear behavior is present in the mean-squared displacement [Fig. 4(a)]. Due to the absence of data in the range $0.5<T<1.0$, it is difficult to unambiguously identify a concave-to-convex crossover in $F_{D D}(q, t)$ by varying temperature [Fig. 6(a)]. Figure 10(a) shows results by varying $q$ at constant $T=0.30$. The trend exhibited by the data, which show a extremely stretched decay at large wave vectors, suggests that such a crossover might be present for higher values of $q$. However, we cannot confirm this point, which remains to be understood. We have not detected the crossover at least up to $q \approx 7 \sigma_{D D}^{-1}$. Beyond that $q$ value the amplitude of the decay is rather small and it is difficult to solve the shape of the curve within statistical noise.

Still, Eq. (7) provides a good description of the decay [Fig. 10(a)], with a common $f_{q}^{\mathrm{c}}$ for different state points [Fig. 10(b)] and $H_{q}$ obeying scaling behavior [Fig. 10(c)]. Surprisingly, the scaling factor is unity within error bars, despite the dynamics at state points $T=0.30$ and $T=0.40$ being significantly different [see Figs. 4(a) and 6(a)].

Due to the complex form of the decay of $F_{D D}(q, t)$ for composition $x_{\text {small }}=0.8$ [Fig. 6(c)], a fit to Eq. (3) or (7) is not possible. Hence, at this composition there are no apparent analogies for small particles with known MCT scenarios.

\section{DISCUSSION}

Results presented in Secs. III and IV exhibit strong dynamic analogies with MCT predictions for state points close 

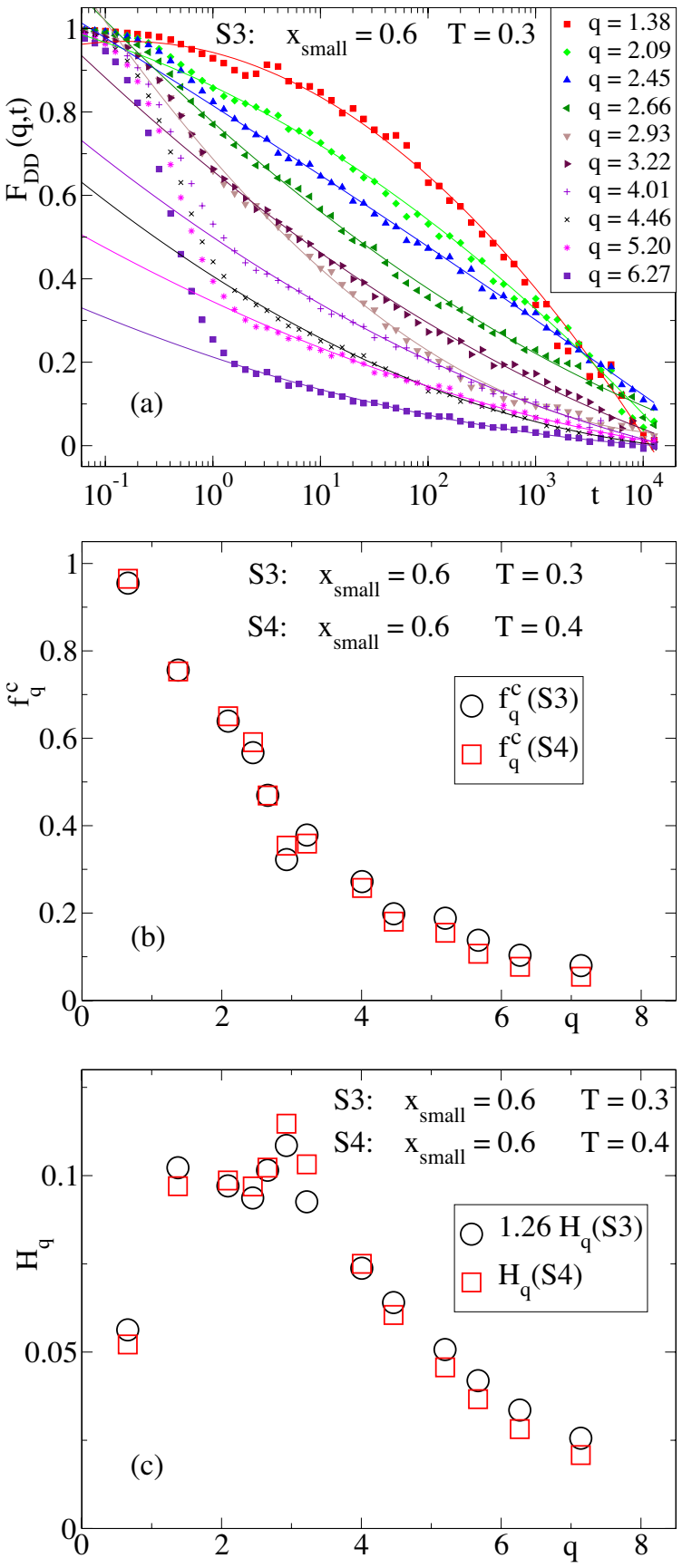

FIG. 9. (Color online) As in Fig. 8 for $F_{D D}(q, t), x_{\text {small }}=0.6$, at the state points $S 3$ and $S 4$. The characteristic times $\tau$ for $S 3$ and $S 4$ are, respectively, 30 and 7.2. Data in panel (a) for the wave vector $q=2.45 \sigma_{D D}^{-1}$ exhibit logarithmic decay over four time decades.

to higher-order transitions $(\lambda=1)$ or, more generally, for $A_{2}$ transitions with exponent parameter $\lambda \lesssim 1$. These analogies must not be understood as a proof of an underlying MCT scenario for the mixture of large and small particles here investigated. An unambiguous answer to this question could only be provided by solving the corresponding MCT equations. As mentioned in the Introduction, theoretical and computational works on the framework of the MCT on mixtures of large and small hard spheres have not reported anomalous relaxation features [23-25]. However, these works have ex-
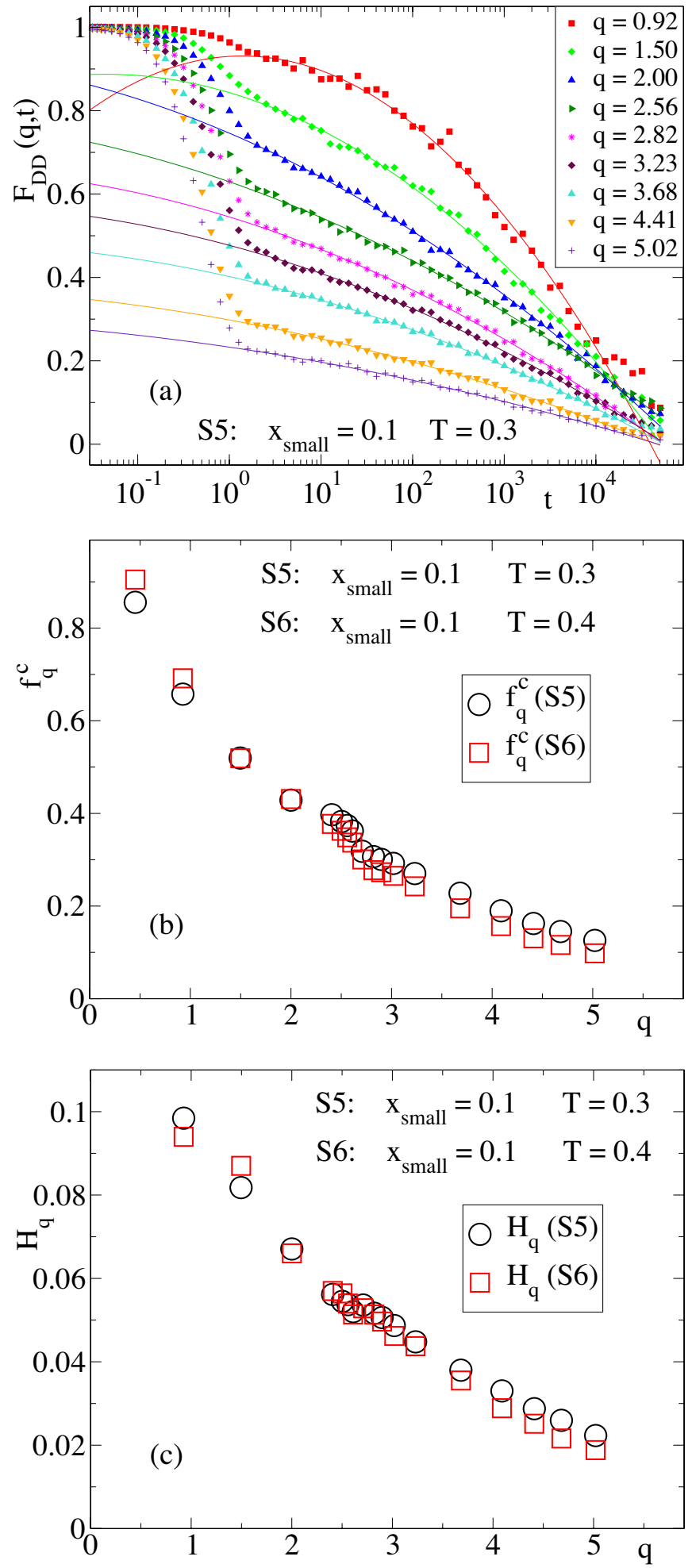

FIG. 10. (Color online) As Fig. 8 for $F_{D D}(q, t), x_{\text {small }}=0.1$, at the state points $S 5$ and $S 6$. The characteristic times $\tau$ for $S 5$ and $S 6$ are, respectively, 360 and 50 .

plored size disparities smaller than the value $\delta=\sigma_{A A} / \sigma_{C C}$ $=\sigma_{B B} / \sigma_{D D}=2.5$ used in this work.

Figure 11 displays typical slabs of the simulation box for mixture compositions $x_{\text {small }}=0.1,0.6$, and 0.8 . The slab thickness is $5 \sigma_{D D}$. On the basis of the particular features 

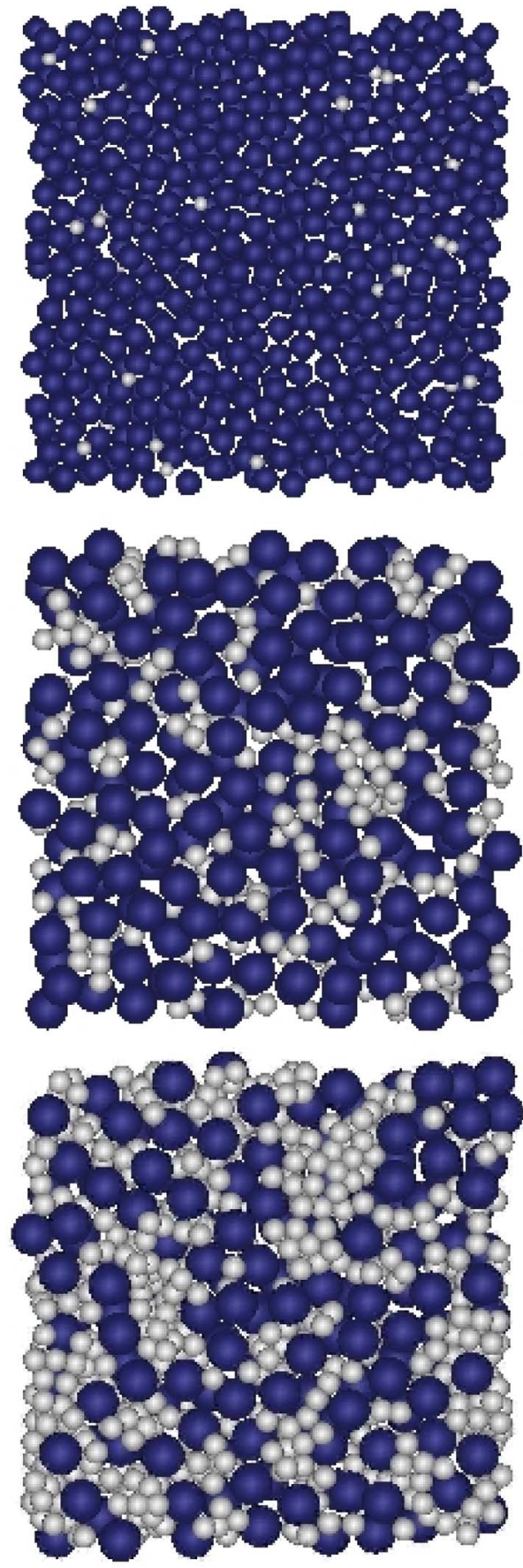

FIG. 11. (Color online) Typical slabs (of thickness $5 \sigma_{D D}$ ) of different configurations at $T=0.30$. From top to down $x_{\text {small }}=0.1$, 0.6, and 0.8. Dark: $A$ and $B$ particles. Light: $C$ and $D$ particles.

displayed by the configuration of small and large particles at the different compositions, next we discuss the observed anomalous relaxation features in terms of competition between different arrest mechanisms.

\section{A. Symmetric mixtures}

For composition $x_{\text {small }}=0.6$, large particles are distributed over the simulation box in a rather homogeneous way, with a weak tendency to form clusters. Small particles fill connected cavities in the slow matrix of large particles, which acts as a confining medium for the formers. As a consequence, competition between bulklike dynamics and confinement occurs for arrest of the small particles. Pure logarithmic decay in $F_{D D}(q, t)$ is observed for $q \sim 2.5 \sigma_{D D}^{-1}$ [Fig. 9(a)]i.e., for wavelengths $\sim 2.5 \sigma_{D D}$ probing the cavity size (Fig. 11 , middle).

Dynamic arrest of large particles is essentially driven by soft-sphere repulsion, as it would be for a system without size disparity, and a consistent description of dynamic features is achieved within the standard $A_{2}$ scenario of the MCT. Still, it must be mentioned that the obtained value of the exponent parameter, $\lambda=0.887$, is significantly higher than typical values $\lambda \sim 0.7$ usually obtained within the $A_{2}$ scenario [2-5]. This high value of $\lambda$ can be interpreted as a signature of the depletion mechanism induced by neighboring small particles, which leads to the observed weak clusterization of large particles. Though only weakly competing with softsphere repulsion, the depletion mechanism yields precursor effects of an incoming higher-order scenario $(\lambda \rightarrow 1)$, as strong stretching for the long-time decay in $F_{\mathrm{AA}}(q, t)$ [Fig. $7(b)]$, or sublinear behavior in the mean-squared displacement [Fig. 3(b)]. These effects are weaker for the composition $x_{\text {small }}=0.3$, yielding a lower exponent parameter $\lambda$ $=0.880$.

\section{B. High concentration of small particles}

For composition $x_{\text {small }}=0.8$, the population of small particles is sufficient to provide an efficient depletion mechanism, yielding a strong clusterization of the large particles (Fig. 11, bottom). Competition between soft-sphere repulsion and depletion leads to anomalous relaxation features for the large particles. Hence, $F_{A A}(q, t)$ shows logarithmic decay for $q \sim 5.6 \sigma_{D D}^{-1}$ [Fig. 8(a)]-i.e., for wavelengths $\sim \sigma_{D D}$ probing the size of the small particles.

Though depletion effects are evidenced by clusterization of large particles, it is worth emphasizing that a direct comparison with results for the effective one-component systems displaying the higher-order MCT scenario [6,7,9,11-14] cannot be made. In the effective one-component systems the small particles are absent and the depletion mechanism is described by an effective short-ranged attraction between the large particles [19]. However, concerning dynamics, the validity of this approximation does not only require a much smaller size, but also a much smaller mass for the small particles [38]. In the case here investigated large and small particles have the same mass. Therefore, a proper proof of the existence of an underlying higher-order MCT scenario can only be provided by solving the MCT equations for the two-component system.

Density-density correlators for small particles at $x_{\text {small }}$ $=0.8$ exhibit a complex decay. An apparent logarithmic decay occurs at long times [Fig. 6(c)]. For $T=0.30$ the onset of this decay occurs at $t \approx 60$, which corresponds to mean displacements $\left\langle\left(\Delta r_{D}\right)^{2}\right\rangle^{1 / 2} \sim 2 \sigma_{D D}$ [Fig. 4(c)]. If logarithmic decay is again interpreted as the result of a competition between bulklike dynamics and confinement, the former 


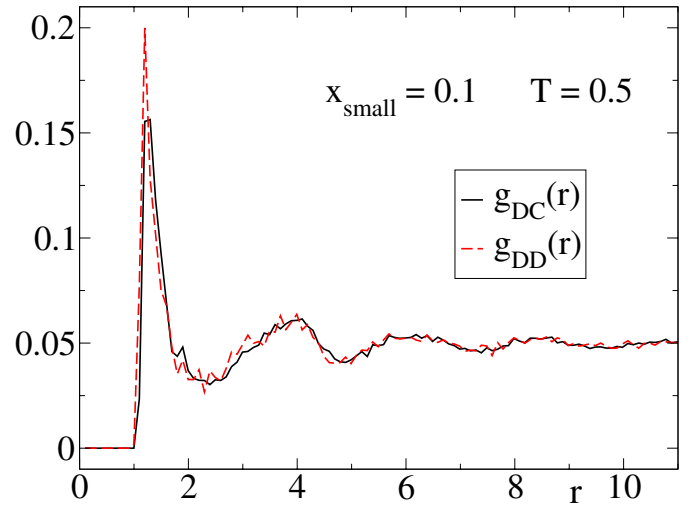

FIG. 12. (Color online) Radial distribution function for $D-C$ and $D-D$ pairs, for $x_{\text {small }}=0.1$ and $T=0.50$.

distance would correspond to the length scale beyond which confinement induced by the large particles affects dynamic arrest of the small particles. For smaller length scales, the only relevant arrest mechanism is bulklike caging induced by neighboring small particles. Hence, for $t \leqslant 60$ small particles do not exhibit clear signatures of anomalous relaxation, as suggested by Figs. 4(c) and 6(c).

\section{Low concentration of small particles}

For $x_{\text {small }}=0.1$, most of the small particles are isolated in the slow matrix of large particles (Fig. 11, top). However, there is also a significant population of pairs of neighboring small particles. We have estimated that about a $7 \%$ of the small particles have a neighboring small particle within a distance $1.3 \sigma_{D D}$. Clusters of three or more neighboring small particles for this interparticle distance are very rare.

The existence of pairs of neighboring small particles yields a sharp peak at distances $\sigma_{D D} \lesssim r \lesssim 2.5 \sigma_{D D}$ for the radial distribution function for $D-C\left[g_{D C}(r)\right]$ and $D-D$ $\left[g_{D D}(r)\right]$ pairs, as shown in Fig. 12. Hence, even for so low populations of small particles, it may be expected that competition between bulklike dynamics and confinement occurs for a significant fraction of particles. In order to test this hypothesis we have calculated mean-squared displacements and density-density correlators for the subset of $D$ particles which initially (i.e., at the time origin for the calculation of both quantities) have at least one neighboring small $(C$ or $D)$ particle within a distance $r<1.3 \sigma_{D D}$. This distance approximately corresponds to the location of the maximum in $g_{D C}(r)$ and $g_{D D}(r)$. Figure 13 shows $\left\langle\left(\Delta r_{D}\right)^{2}\right\rangle$ and $F_{D D}(q, t)$, computed for the former subset of $D$ particles and compared with the corresponding quantities computed for all the $D$ particles [previously shown in Figs. 4(a) and 10(a)]. Relaxation of $D$ particles having neighboring small particles is, at intermediate times, faster than the average over all $D$ particles, which is approached only for very long times corresponding to the onset of the linear diffusive regime.

Differently from the average over all $D$ particles, the subset initially having neighboring small particles does exhibit anomalous relaxation features. Mean-squared displacements display sublinear behavior over two time decades. The cor-
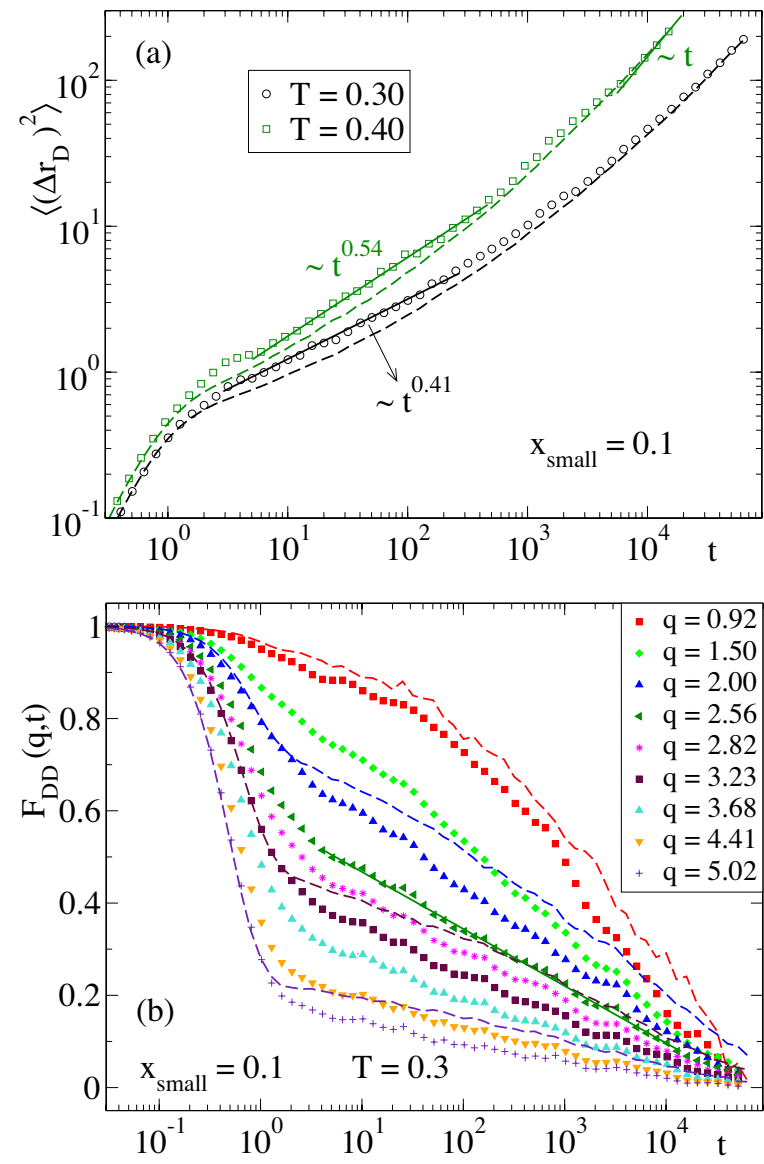

FIG. 13. (Color online) Symbols: for composition $x_{\text {small }}=0.1$, mean-squared displacement [panel (a)] and density-density correlator [panel (b)] for the subset of $D$ particles which initially have at least one neighboring small particle within a distance $r$ $<1.3 \sigma_{D D}$. Panel (a) shows data for $T=0.30$ and 0.40 . Dashed curves are the corresponding data by averaging over all the $D$ particles. Solid lines indicate linear or sublinear behavior. Panel (b) shows data for different $q$ values at $T=0.30$. Dashed curves are data by averaging over all the $D$ particles (from top to down, $q \sigma_{D D}$ $=0.92,2.00,3.23$, and 5.02). The solid line indicates logarithmic relaxation.

responding exponent decreases with decreasing temperature [Fig. 13(a)]. Density-density correlators show a concave-toconvex crossover [Fig. 13(b)]. Logarithmic relaxation is observed for wave vectors $q \sim 2.5 \sigma_{D D}^{-1}$ [Fig. 13(a)]—i.e., for length scales $\sim 2.5 \sigma_{D D}$ which probe the first minimum in $g_{D C}(r)$ and $g_{D D}(r)$.

We have also computed $\left\langle\left(\Delta r_{D}\right)^{2}\right\rangle$ and $F_{D D}(q, t)$ for the subset of $D$ particles which initially do not have any neighboring small particle within a distance $r<4.5 \sigma_{D D}$-i.e., for initially isolated $D$ particles. The results for this subset (not shown) are hardly distinguishable from those averaged over all $D$ particles and reported in Figs. 4(a) and 10(a). Hence, initially isolated $D$ particles do not show anomalous relaxation features as sublinear behavior for mean-squared displacements or concave-to-convex and logarithmic decay for density-density correlators. 


\section{CONCLUSIONS}

We have carried out simulations on a mixture of large and small particles. The slow dynamics have been investigated for a broad range of temperature and mixture composition. The introduction of a significant size disparity yields very different time scales for large and small particles, reaching differences of from two to three decades in diffusivity for the lowest investigated temperatures. This model exhibits nonconventional relaxation features. Mean-squared displacements display sublinear behavior at intermediate times. The exponent for the corresponding power law decreases by decreasing the temperature. By varying the temperature or wave vector, a concave-to-convex crossover is obtained for the shape of the decay of density-density correlators. At some intermediate point of this crossover, the decay is purely logarithmic.

These anomalous relaxation features, which are observed over time intervals extending up to four decades, strongly resemble predictions of the mode coupling theory for state points close to higher-order transitions, which originate from the competition between different mechanisms for dynamic arrest. By varying the mixture composition, anomalous relaxation is displayed by both the large and small particles. For the large particles we suggest competition between softsphere repulsion and depletion effects induced by neighboring small particles. For the small particles we suggest competition between bulklike dynamics and confinement, respectively, induced by neighboring small particles and by the slow matrix of large particles.

Simulation results reported here do not constitute a rigorous proof of an inherent MCT scenario as the one described above. A proper answer to this question can only be provided by solving the corresponding MCT equations. However, the highly nontrivial observed analogies suggest one to consider it as a plausible hypothesis. This view is also supported by MCT theoretical calculations in a mixture of fixed and mobile hard spheres $[26,27]$, which explicitly report a higherorder MCT transition [39].

Though being beyond the scope of this article, the observation of confinement effects also suggests to test a more speculative hypothesis: a MCT scenario for a liquid of spheres in fractal dimensions as the origin of the observed relaxation features for the small particles. To our knowledge no MCT calculations of mean-squared displacements or dynamic correlators are available for the latter or similar systems.

\section{ACKNOWLEDGMENTS}

We thank E. Zaccarelli, F. Sciortino, T. Voigtmann, V. Krakoviack, and J. Horbach for useful discussions. We acknowledge support from Projects Nos. NMP3-CT-2004502235 (SoftComp), MAT2004-01017 (Spain), and 206.21513568/2001 (GV-UPV/EHU Spain).
[1] W. Götze and L. Sjögren, Rep. Prog. Phys. 55, 241 (1992).

[2] W. Götze, J. Phys.: Condens. Matter 11, A1 (1999).

[3] W. Kob and H. C. Andersen, Phys. Rev. Lett. 73, 1376 (1994); Phys. Rev. E 51, 4626 (1995); 52, 4134 (1995).

[4] C. Bennemann, W. Paul, K. Binder, and B. Dunweg, Phys. Rev. E 57, 843 (1998); C. Bennemann, J. Baschnagel, and W. Paul, Eur. Phys. J. B 10, 323 (1999).

[5] M. Aichele and J. Baschnagel, Eur. Phys. J. B 5, 229 (2001); 5, 245 (2001).

[6] F. Sciortino, P. Tartaglia, and E. Zaccarelli, Phys. Rev. Lett. 91, 268301 (2003).

[7] E. Zaccarelli, G. Foffi, K. A. Dawson, S. V. Buldyrev, F. Sciortino, and P. Tartaglia, Phys. Rev. E 66, 041402 (2002).

[8] A. J. Moreno and J. Colmenero, J. Chem. Phys. 124, 184906 (2006).

[9] M. Sperl, Phys. Rev. E 68, 031405 (2003).

[10] S. P. Das, Rev. Mod. Phys. 76, 785 (2004).

[11] L. Fabbian, W. Götze, F. Sciortino, P. Tartaglia, and F. Thiery, Phys. Rev. E 59, R1347 (1999).

[12] J. Bergenholtz and M. Fuchs, Phys. Rev. E 59, 5706 (1999).

[13] K. Dawson, G. Foffi, M. Fuchs, W. Götze, F. Sciortino, M. Sperl, P. Tartaglia, Th. Voigtmann, and E. Zaccarelli, Phys. Rev. E 63, 011401 (2000).

[14] W. Götze and M. Sperl, J. Phys.: Condens. Matter 15, S869 (2003).

[15] A. M. Puertas, M. Fuchs, and M. E. Cates, Phys. Rev. Lett. 88, 098301 (2002).

[16] F. Mallamace, P. Gambadauro, N. Micali, P. Tartaglia, C. Liao, and S.-H. Chen, Phys. Rev. Lett. 84, 5431 (2000).

[17] K. N. Pham, A. M. Puertas, J. Bergenholtz, S. U. Egelhaaf, A. Moussaid, P. N. Pusey, A. B. Schofield, M. E. Cates, M. Fuchs, and W. C. K. Poon, Science 296, 104 (2002).

[18] T. Eckert and E. Bartsch, Phys. Rev. Lett. 89, 125701 (2002).

[19] C. N. Likos, Phys. Rep. 348, 267 (2001).

[20] A recent computational work by Zaccarelli et al. [21] has reported anomalous relaxation features for a short-ranged attractive colloid with a constraint in the maximum number of bonds for each particle (note that the standard case is unconstrained). Hard-sphere repulsion and reversible gelation of a bonded network have been suggested as competing arrest mechanisms for this system.

[21] E. Zaccarelli, I. Saika-Voivod, S. V. Buldyrev, A. J. Moreno, P. Tartaglia, and F. Sciortino, J. Chem. Phys. 124, 124908 (2006).

[22] A. C. Genix, A. Arbe, F. Alvarez, J. Colmenero, L. Willner, and D. Richter, Phys. Rev. E 72, 031808 (2005); J. Colmenero et al. (unpublished).

[23] W. Götze and Th. Voigtmann, Phys. Rev. E 67, 021502 (2003).

[24] G. Foffi, W. Götze, F. Sciortino, P. Tartaglia, and Th. Voigtmann, Phys. Rev. Lett. 91, 085701 (2003).

[25] G. Foffi, W. Götze, F. Sciortino, P. Tartaglia, and Th. Voigtmann, Phys. Rev. E 69, 011505 (2004).

[26] V. Krakoviack, Phys. Rev. Lett. 94, 065703 (2005).

[27] V. Krakoviack, J. Phys.: Condens. Matter 17, S3565 (2005).

[28] The slow dynamics of mobile particles in matrixes of static 
particles have been investigated within the MCT framework in several simulation studies [29]. In these works the relative contribution of the static particles to the total packing fraction is about a $30 \%$. Though quantitive deviations from the bulk counterpart are obtained for the mobile particles, no evidence of anomalous relaxation features is observed and a description in terms of the standard MCT scenario is possible. According to the theoretical work in Ref. [26], the higher-order MCT transition would be located at a relative contribution of the static particles of about $40 \%$. It is well known that MCT usually overestimates the location of the transition point. Presumably, computational tests on the system investigated in Ref. [26] would provide a higher value for the relative contribution of static particles and therefore far from that used in Ref. [29]. Though no direct comparison is possible with the system here investigated (large particles are slower than small ones, not static), we mention that for the highest value of $x_{\text {small }}=0.8$ here investigated at size disparity $\delta=2.5$ the relative contribution of large particles to the total packing fraction is $80 \%$.

[29] P. Gallo, R. Pellarin, and M. Rovere, Europhys. Lett. 57, 212 (2002); Phys. Rev. E 67, 041202 (2003); 68, 061209 (2003).

[30] We have observed some partial crystallization of the large component for the composition $x_{\text {small }}=0.1$ at temperature $T$ $\sim 0.70$. No crystallization effects are observed at this concentration for the other investigated temperatures $T=1.0$ and $T$ $\leq 0.50$. For the latter range, the matrix of large particles, which is quenched from high temperature, is out of equilibrium (see Sec. III). Partial crystallization would presumably occur for sufficiently long computional times.

[31] D. Frenkel and B. Smit, Understanding Molecular Simulation (Academic Press, San Diego, 1996).

[32] A MCT transition of order $n, A_{n}$, is characterized by $n$ solutions of the critical nonergodicity parameter, only the highest value being physical.
[33] W. Götze and R. Haussmann, Z. Phys. B: Condens. Matter 72, 403 (1988).

[34] W. Götze and M. Sperl, Phys. Rev. E 66, 011405 (2002).

[35] A correction to $f_{q}^{\mathrm{c}}$, of the order of the distance to the transition point, is neglected in Eq. (7).

[36] For low- $q$ values, the fits in Fig. 8(a) only reproduce a reduced time window of the simulation results. However, this is not necessarily a failure of MCT predictions in this $q$ range. A similar example is reported in Ref. [25] for a binary mixture of moderate size disparity. As explained there, if one is not extremely close to the transition point, it can accidentaly happen for some $q$ values that the third-order term of the series expansion (which is usually neglected in fitting procedures) is not actually negligible as compared to the second-order term. Hence a fit to a second-order expansion as Eq. (7) would provide a good description of the data only for a reduced time interval. One could fit data in Fig. 8(a) by adding a third-order term, but the fitting parameters would be strongly coupled and their so-obtained values would not be reliable. Anyway, a proper answer to this point can only be provided by solving the corresponding MCT equations.

[37] Strictly speaking, MCT calculations for one-component shortranged attractive colloids predict a weak modulation of $f_{q}^{\mathrm{c}}$ at small wave vectors $[9,13]$. This modulation is not observed in computational investigations [6]. This absence originates from mixing effects resulting from the introduction in those works of a small size disparity to avoid crystallization [9].

[38] E. Zaccarelli, H. Löwen, P. P. F. Wessels, F. Sciortino, P. Tartaglia, and C. N. Likos, Phys. Rev. Lett. 92, 225703 (2004).

[39] Preliminary results by using the size disparity as a control parameter seem to display further analogies with the dynamic picture provided in [26,27]. See A. J. Moreno and J. Colmenero, e-print cond-mat/0604171. 